\title{
Parameterized Model Order Reduction with Guaranteed Passivity for PEEC Circuit Analysis
}

\author{
Francesco Ferranti, Tom Dhaene, Luc Knockaert \\ Department of Information Technology (INTEC) \\ Ghent University \\ IBBT, Sint Pietersnieuwstraat 41, 9000, Ghent, Belgium \\ Email: francesco.ferranti@intec.ugent.be; \\ tom.dhaene@intec.ugent.be; luc.knockaert@intec.ugent.be
}

\author{
Giulio Antonini \\ Dipartimento di Ingegneria Elettrica e dell'Informazione \\ Università degli Studi dell'Aquila \\ Via G. Gronchi 18, 67040, L'Aquila, Italy \\ Email: giulio.antonini@univaq.it
}

\begin{abstract}
We present a novel parameterized model order reduction technique applicable to the Partial Element Equivalent Circuit analysis that provides parametric reduced order models, stable and passive by construction, over a user defined design space. We treat the construction of parametric reduced order models on scattered design space grids. This new parameterized model order reduction technique is based on the hybridization of traditional passivity-preserving model order reduction methods and interpolation schemes based on a class of positive interpolation operators, in order to guarantee overall stability and passivity of the parametric reduced order model. Pertinent numerical examples validate the proposed approach.
\end{abstract}

\section{INTRODUCTION}

The analysis and design of complex high-speed systems require the use of 3-D electromagnetic (EM) methods [1][3]. These EM methods usually results in very large systems of equations which are often prohibitively expensive to solve and model order reduction (MOR) techniques are therefore needed to reduce the resulting high model complexity and computational cost of the simulations [4], [5]. The Partial Element Equivalent Circuit (PEEC) method has gained increasing popularity among electromagnetic compatibility engineers, since it is able to transform the EM system under examination into a passive RLC equivalent circuit [2], [6], [7]. Nonlinear circuit devices, such as drivers and receivers, are usually connected with PEEC equivalent circuits using a time domain circuit simulator (e.g. SPICE). However, inclusion of the PEEC model directly into a circuit simulator may be computationally intractable for complex structures, because the number of circuit elements can be in the tens of thousands. MOR techniques become necessary to reduce the size of a PEEC model [4], [5].

Optimization and design space exploration are usually performed during a typical design process that consequently requires multiple frequency-domain simulations for different design parameter values (e.g. layout features). Performing these design activities by means of EM simulations may often be not feasible due to the high computational complexity. Traditional MOR techniques perform model reduction only with respect to frequency and a new reduced model has to be generated each time a design parameter is modified, thereby reducing the CPU efficiency. Parameterized model order reduction (PMOR) methods are therefore needed to efficiently perform these design activities. PMOR techniques can reduce large systems of equations with respect to frequency and other design parameters and finally build a parameterized reduced order model.

A number of PMOR methods have been developed. Some PMOR techniques are based on statistical performance analysis [8], [9]. The multiparameter moment-matching methods presented in [10], [11] use a subspace projection approach and guarantee the passivity. However, the resulting reduced models usually suffer from oversize when the number of moments to match is high, either because high accuracy (order) is required or because the number of parameters is large. The Parameterized Interconnect Macromodeling via a twodirectional Arnoldi process (PIMTAP) algorithm presented in [12] is numerically stable, preserves the passivity of parameterized RLC networks, but, such as all multiparameter momentmatching based PMOR techniques, it is suitable only to a lowdimensional design space.

This paper proposes a PMOR method applicable to PEEC analysis that provides parametric reduced order models, stable and passive by construction, over the design space of interest. We treat the construction of parametric reduced order models on scattered design space grids that are more flexible than fully filled grids and allow the use of adaptive sampling schemes that can be useful to select a minimal and well-distributed set of points in the design space. This new PMOR technique combines traditional passivity-preserving MOR methods and interpolation schemes based on a class of positive interpolation operators [13], in order to guarantee overall stability and passivity of the parametric reduced order model. The hybridization of PEEC models, MOR methods and interpolation schemes is used in the proposed PMOR methods. Recently, a method has been proposed in [14], based on an efficient and reliable combination of rational identification and positive interpolation schemes to build parameterized macromodels, stable and passive by construction, over the design space of interest, starting from multivariate data samples of the inputoutput system behavior and not from system equations as in all PMOR techniques previously discussed. 
This paper is organized as follows. Section II describes the modified nodal analysis (MNA) equations of the PEEC method. Section III describes the proposed PMOR method. Finally, some pertinent numerical examples are presented in Section IV, validating the proposed technique.

\section{PEEC FORMULATION}

The PEEC method [2] stems from the integral equation form of Maxwell's equations. With respect to other EM methods, it is worth pointing out its capability to provide a circuit interpretation of the EFIE equation, thus allowing to handle complex problems involving both circuits and electromagnetic fields [2], [7], [15]-[17]. In what follows, we describe a quasistatic PEEC formulation [2] that approximates the full-wave PEEC approach [15].

The original approach [2], requires the discretization of volumes and surfaces into elementary regions, hexahedra and patches respectively [17], over which the current and charge densities are expanded into a series of pulse basis functions. Both conductors and dielectrics can be easily modeled. Conductive elementary volumes are modeled by their ohmic resistance, while polarization effects are described by means of the excess capacitance which is placed in series to the partial inductance of each elementary volume cell. An example of PEEC circuit electrical quantities is illustrated, in the Laplace domain, in Fig. 1 where the current controlled voltage sources $s L_{p, i j} I_{j}$ and the current controlled current sources $I_{c c i}$ model the magnetic and electric field coupling, respectively.

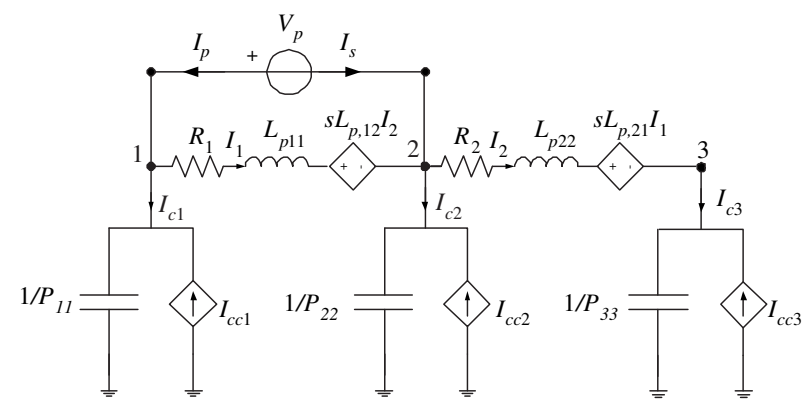

Fig. 1. Illustration of PEEC circuit electrical quantities for a conductor elementary cell.

Let us assume that the meshing process of conductors and dielectrics has generated $n_{i}$ volume cells where currents flow and $n_{n}$ surface cells where charge is located; the resultant number of elementary cells of conductors and dielectrics is $n_{c}$ and $n_{d}$, respectively and that of electrical nodes is $n_{n}$. Furthermore, let us assume to be interested in generating an admittance representation $\mathbf{Y}(s)$ having $n_{p}$ output currents $\mathbf{i}_{p}(t)$ under voltage excitation $\mathbf{v}_{p}(t)$. If the MNA approach [18] is used, the global number of unknowns is $n_{u}=n_{i}+n_{d}+n_{n}+n_{p}$ and an admittance representation of the PEEC circuit is obtained

$$
\begin{aligned}
\mathbf{C} \frac{d \mathbf{x}(t)}{d t} & =-\mathbf{G x}(t)+\mathbf{B u}(t) \\
\mathbf{i}_{p}(t) & =\mathbf{L}^{T} \mathbf{x}(t)
\end{aligned}
$$

where $\mathbf{x}(t) \in \Re^{n_{u} \times 1}, \mathbf{C} \in \Re^{n_{u} \times n_{u}}, \mathbf{G} \in \Re^{n_{u} \times n_{u}}$ and $\mathbf{B}=$ $\mathbf{L}, \mathbf{B} \in \Re^{n_{u} \times n_{p}}$. This is an admittance $n_{p}$-port formulation $\mathbf{Y}(s)=\mathbf{L}^{T}(s \mathbf{C}+\mathbf{G})^{-1} \mathbf{B}$, whereby the only sources are the voltage sources at the $n_{p}$-port nodes. If we consider $\mathrm{N}$ design parameters $\boldsymbol{g}=\left(g^{(1)}, \ldots, g^{(\mathrm{N})}\right)$ in addition to frequency, the equations (1a)-(1b) become

$$
\begin{aligned}
\mathbf{C}(\boldsymbol{g}) \frac{d \mathbf{x}(t, \boldsymbol{g})}{d t} & =-\mathbf{G}(\boldsymbol{g}) \mathbf{x}(t, \boldsymbol{g})+\mathbf{B}(\boldsymbol{g}) \mathbf{u}(t) \\
\mathbf{i}_{p}(t, \boldsymbol{g}) & =\mathbf{L}(\boldsymbol{g})^{T} \mathbf{x}(t, \boldsymbol{g})
\end{aligned}
$$

\section{A. Properties of PEEC formulation}

Stability and passivity are fundamental properties for a model that is used in a simulator that performs transient analysis. While a passive system is also stable, the reverse is not necessarily true [19]. Passivity refers to the property of systems that cannot generate more energy than they absorb through their electrical ports. When a passive system is terminated on any set of arbitrary passive loads, none of them will cause the system to become unstable. A linear network described by admittance matrix $\mathbf{Y}(s)$ is passive (or positivereal) if [20]:

1) $\mathbf{Y}\left(s^{*}\right)=\mathbf{Y}^{*}(s)$ for all $s$, where "*” is the complex conjugate operator.

2) $\mathbf{Y}(s)$ is analytic in $\Re e(s)>0$.

3) $\mathbf{Y}(s)$ is a positive-real matrix, i.e. : $\mathbf{z}^{* T}\left(\mathbf{Y}(s)+\mathbf{Y}^{T}\left(s^{*}\right)\right) \mathbf{z} \geq 0 ; \forall s: \Re e(s)>0$ and any arbitrary vector $\mathbf{z}$.

Since the matrices $\mathbf{P}^{-1}, \mathbf{L}_{p}, \mathbf{C}_{d}, \mathbf{R}$ are symmetric nonnegative definite matrices by construction, it is straightforward to prove that the matrices $\mathbf{C}, \mathbf{G}$ satisfy the following properties

$$
\begin{aligned}
& \mathbf{C}=\mathbf{C}^{T} \geq 0 \\
& \mathbf{G}+\mathbf{G}^{T} \geq 0
\end{aligned}
$$

The properties of the PEEC matrices $\mathbf{B}=\mathbf{L}, \mathbf{C}=\mathbf{C}^{T} \geq$ $0, \mathbf{G}+\mathbf{G}^{T} \geq 0$ ensure the passivity of the PEEC admittance model $\mathbf{Y}(s)=\mathbf{L}^{T}(s \mathbf{C}+\mathbf{G})^{-1} \mathbf{B}[21]$ and allow to exploit the passivity-preserving capability of the Laguerre-based MOR algorithm.

\section{PARAMETERIZED MODEL ORDER REDUCTION}

In this section we describe a PMOR technique that is able to generate reduced order models as a function of frequency and $\mathrm{N}$ design parameters $\boldsymbol{g}=\left(g^{(1)}, \ldots, g^{(\mathrm{N})}\right)$, such as the layout features of a circuit (e.g. lengths, widths,...) or the substrate parameters (e.g. thickness, dielectric constant, losses,...). The PMOR method aims at accurately approximating the original scalable system (having a high complexity) with a reduced scalable system (having a low complexity) by capturing the 
behavior of the original system with respect to frequency and other design parameters. Stability and passivity of the parametric reduced model are guaranteed over the entire design space of interest by construction. The different steps of the proposed PMOR method are shown by a flowchart in Fig. 2.

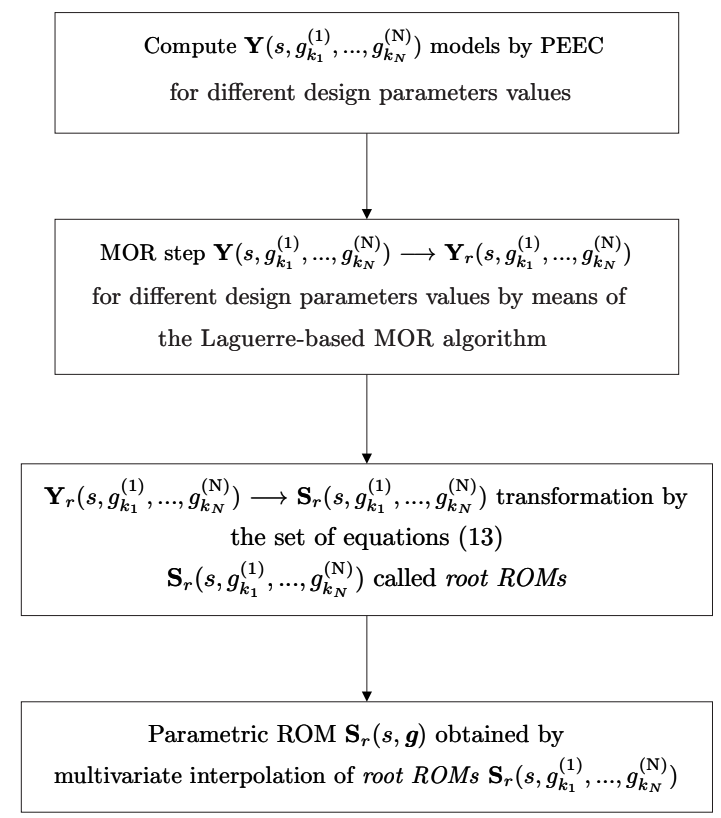

Fig. 2. Flowchart of the proposed PMOR method.

\section{A. Root ROMs}

As first step, the proposed PMOR technique computes a set of stable and passive reduced order models of the PEEC admittance matrix $\mathbf{Y}(s, \boldsymbol{g})$ using the Laguerre-based algorithm for different design parameters values in the design space $\left\{\boldsymbol{g}_{k}\right\}_{k=1}^{K_{t o t}}$. The $\mathbf{S}$-representation is used to describe the broadband frequency behavior of microwave systems, since at microwave frequencies, the $\mathbf{Y}$-representation cannot be accurately measured because the required short-circuit tests are difficult to achieve over a broad range of frequencies. Consequently, a transformation from $\mathbf{Y}$-representation into S-representation is performed, while preserving stability and passivity by the procedure described in Section III-B. In this paper we refer to these initial S-reduced order models as root ROMs. This initial step allows the separation of frequency from the other parameters, in other words frequency is treated as a special parameter. Every root ROM is related to a specific point in the design space. The construction of the root ROMs results in a family of univariate reduced models related to a specific set of points in the design space. The design space $\mathcal{D}(\boldsymbol{g})$ is defined as the parameter space $\mathcal{P}(s, \boldsymbol{g})$ without frequency. The parameter space $\mathcal{P}(s, \boldsymbol{g})$ contains all parameters $(s, \boldsymbol{g})$. If the parameter space is $\mathrm{N}$-dimensional, the design space is $(\mathrm{N}-1)$-dimensional.

\section{B. $Y$-S transformation}

The definition of the S-representation and its relation to the other system representations depend on the reference impedance at each port $\mathrm{Z}_{0, i}$, that in practice is often chosen equal to $50 \Omega$. Let $\mathbf{Z}_{0}$ be a real diagonal matrix such that $\mathbf{Z}_{0}(i, i)=\mathbf{Z}_{0, i}$, then the $\mathbf{S}$-representation is related to the $\mathbf{Y}$ representation by

$$
\mathbf{S}(s)=\left(\mathbf{I}-\mathbf{Z}_{0}^{1 / 2} \mathbf{Y}(s) \mathbf{Z}_{0}^{1 / 2}\right)\left(\mathbf{I}+\mathbf{Z}_{0}^{1 / 2} \mathbf{Y}(s) \mathbf{Z}_{0}^{1 / 2}\right)^{-1}
$$

It is possible to obtain a descriptor form $\mathbf{S}_{\mathbf{r}}(s, \boldsymbol{g})=\left[\widetilde{\mathbf{C}_{\mathbf{r}}}(\boldsymbol{g}), \widetilde{\mathbf{G}_{\mathbf{r}}}(\boldsymbol{g}), \widetilde{\mathbf{B}_{\mathbf{r}}}, \widetilde{\mathbf{L}_{\mathbf{r}}}(\boldsymbol{g}), \widetilde{\mathbf{D}_{\mathbf{r}}}(\boldsymbol{g})\right]$ from a descriptor form of the reduced order model $\mathbf{Y}_{\mathbf{r}}(s, \boldsymbol{g})=\left[\mathbf{C}_{\mathbf{r}}(\boldsymbol{g}), \mathbf{G}_{\mathbf{r}}(\boldsymbol{g}), \mathbf{B}_{\mathbf{r}}(\boldsymbol{g}), \mathbf{L}_{\mathbf{r}}(\boldsymbol{g})\right]$ obtained by means of the Laguerre-based MOR algorithm, using the following equations [22]

$$
\begin{aligned}
& \widetilde{\mathbf{C}_{\mathbf{r}}}(\boldsymbol{g})=\mathbf{C}_{\mathbf{r}}(\boldsymbol{g}) \\
& \widetilde{\mathbf{G}_{\mathbf{r}}}(\boldsymbol{g})=\mathbf{G}_{\mathbf{r}}(\boldsymbol{g})+\mathbf{B}_{\mathbf{r}}(\boldsymbol{g}) \mathbf{Z}_{0} \mathbf{L}_{\mathbf{r}}(\boldsymbol{g})^{T} \\
& \widetilde{\mathbf{B}_{\mathbf{r}}}(\boldsymbol{g})=\sqrt{2} \mathbf{B}_{\mathbf{r}}(\boldsymbol{g}) \mathbf{Z}_{0}^{1 / 2} \\
& \widetilde{\mathbf{L}_{\mathbf{r}}}(\boldsymbol{g})=-\widetilde{\mathbf{B}_{\mathbf{r}}}(\boldsymbol{g}) \\
& \widetilde{\mathbf{D}_{\mathbf{r}}}(\boldsymbol{g})=\mathbf{I}
\end{aligned}
$$

We note that the transfer function $\mathbf{Y}(s)$ is positive-real if and only if $\mathbf{S}(s)$ is bounded-real [20], i.e.:

1) $\mathbf{S}\left(s^{*}\right)=\mathbf{S}^{*}(s)$ for all $s$, where "*" is the complex conjugate operator.

2) $\mathbf{S}(s)$ is analytic in $\Re e(s)>0$.

3) $\mathbf{I}-\mathbf{S}^{T}\left(s^{*}\right) \mathbf{S}(s) \geq 0 ; \forall s: \Re e(s)>0$.

The bounded-realness property represents the passivity property for systems described by scattering parameters. Once a passive and stable $\mathbf{Y}$-reduced model is obtained by means of the Laguerre-based MOR technique, a $\mathbf{Y}$-S transformation is performed using the set of equations (5), which results in a reduced S-representation that is still stable and passive.

\section{C. $(N+1)-D$ PMOR}

Once a set of root ROMs is available, the next step of the PMOR algorithm is focused on gluing together the root ROMs by a multivariate interpolation scheme to obtain a parametric reduced model $\mathbf{S}_{r}(s, \boldsymbol{g})$ that preserves stability and passivity over the entire design space. Multivariate interpolation can be realized by means of tensor product [23] or tessellation [24] methods. Tensor product multivariate interpolation methods require that the data points are distributed on a fully filled, but not necessarily equidistant, rectangular grid, while tessellationbased multivariate interpolation methods can handle scattered or irregularly distributed data points. In this paper, we treat the scattered design space grids that are more flexible than fully filled grids and allow the use of adaptive sampling schemes that can be useful to select a minimal and well-distributed set of points in the design space. We propose an interpolation scheme able to cope with scattered design space grids. 
Multivariate simplicial interpolation: Before performing the multivariate interpolation process, the design space is divided into cells using simplices [24]. In 2-D this process is called triangulation, while in higher dimensions it is called tessellation. A simplex, or N-simplex, is the N-D analogue of a triangle in 2-D and a tetrahedron in 3-D. A simplex in $\mathrm{N}$ dimensions has $\mathrm{N}+1$ vertices. For each data distribution many tessellations can be constructed. The minimal requirement is that the simplices do not overlap, and that there are no holes. Delaunay tessellation [24] is a well-known tessellation technique stemming from computational geometry. It is used in different fields such as mesh generation, surface reconstruction, molecular modeling and tessellation of solid shapes. Delaunay tessellation in an $\mathrm{N}$-dimensional space is a spacefilling aggregate of simplices and can be performed using standard algorithms [25]. We indicate a simplex region of the design space as $\Omega_{i}, i=1, \ldots, \mathrm{P}$ and the corresponding $\mathrm{N}+1$ vertices as $\boldsymbol{g}_{k}^{\Omega_{i}}, k=1, \ldots, \mathrm{N}+1$. Once the tessellation of the design space is accomplished, a tessellation-based linear interpolation (TLI) is used to build a parametric reduced order model. TLI performs a linear interpolation inside a simplex using barycentric coordinates [26] as interpolation kernels and it is therefore a local method. If the $\mathrm{N}$-dimensional volume of the simplex does not vanish, i.e., it is non-degenerate, any point enclosed by a simplex can be expressed uniquely as a linear combination of the $\mathrm{N}+1$ simplex vertices. A parametric reduced model can be written as:

$$
\mathbf{S}_{r}(s, \boldsymbol{g})=\sum_{k=1}^{\mathrm{N}+1} \mathbf{S}_{r}\left(s, \boldsymbol{g}_{k}^{\Omega_{i}}\right) \ell_{k}^{\Omega_{i}}(\boldsymbol{g})
$$

where $\Omega_{i}$ is the simplex that contains the point $\boldsymbol{g}$ and the barycentric coordinates $\ell_{k}^{\Omega_{i}}(\boldsymbol{g})$ satisfy the following properties

$$
\begin{aligned}
& 0 \leq \ell_{k}^{\Omega_{i}}(\boldsymbol{g}) \leq 1 \\
& \ell_{k}^{\Omega_{i}}\left(\boldsymbol{g}_{i}^{\Omega_{i}}\right)=\delta_{k, i} \\
& \sum_{k=1}^{N+1} \ell_{k}^{\Omega_{i}}(\boldsymbol{g})=1
\end{aligned}
$$

We remark that the interpolation process is local, because the parametric reduced model $\mathbf{S}_{r}(s, \boldsymbol{g})$ at a specific point $\widehat{\boldsymbol{g}}$ in the design space $\mathcal{D}(\boldsymbol{g})$ only depends on the $\mathrm{N}+1$ root $R O M s$ at the vertices of the simplex that contains the point $\widehat{g}$. The TLI method belongs to the general class of positive interpolation schemes [13]. Stability is automatically preserved in (6), since it is a weighted sum of stable rational models of $s$. The proof of the passivity-preserving property of the proposed PMOR scheme over the entire design space is given in Section III-D. We note that the interpolation kernels we propose only depend on the design space grid points and their computation does not require the solution of a linear system to impose an interpolation constraint. In the bivariate $(s, g)$ case, the interpolation scheme boils down to piecewise linear interpolation. The bivariate reduced model we adopt can be written as

$$
\mathbf{S}_{r}(s, g)=\sum_{k=1}^{\mathrm{K}_{1}} \mathbf{S}_{r}\left(s, g_{k}\right) \ell_{k}(g)
$$

where $\mathrm{K}_{1}$ is the number of the root ROMs, and the interpolation kernels $\ell_{k}(g)$ satisfy conditions (7)-(9).

The proposed PMOR technique is able to deal with scattered design space grids, it is general and any interpolation scheme that leads to a parametric reduced model composed of a weighted sum of root ROMs with weights satisfying (7)-(9) can be used.

\section{Passivity-Preserving Interpolation}

In this section we prove that the proposed PMOR method preserves passivity over the entire design space. Concerning the root ROMs, we have already proven in Section III-B that all three bounded-realness conditions are satisfied. Condition 1) is preserved in (10) and the proposed multivariate extension (6), since they are weighted sums with real nonnegative weights of systems respecting this first condition. Condition 2) is preserved in (10),(6), since they are weighted sums of stable rational reduced models of $s$. Condition 3 ) is equivalent to $\|\mathbf{S}(s)\|_{\infty} \leq 1\left(\mathbf{H}_{\infty}\right.$ norm $)$ [27], i.e., the largest singular value of $\mathbf{S}(s)$ does not exceed one in the right-half $s$-plane. Using this equivalent condition, in the bivariate case we can write

$$
\left\|\mathbf{S}_{r}(s, g)\right\|_{\infty} \leq \sum_{k=1}^{K_{1}}\left\|\mathbf{S}_{r}\left(s, g_{k}\right)\right\|_{\infty} \ell_{k}(g) \leq \sum_{k=1}^{K_{1}} \ell_{k}(g)=1
$$

Similar results are obtained for the proposed multivariate case (6), so condition 3) is satisfied by construction using our PMOR method. We have demonstrated that all three bounded-realness conditions are preserved in the novel PMOR algorithm, using the sufficient conditions (7)-(9) related to the interpolation kernels.

\section{NUMERICAL RESULTS}

\section{3-D example: Multiconductor system with variable separation}

A multiconductor system composed of six conductors with a length $\ell=2 \mathrm{~cm}$, a width $W=1 \mathrm{~mm}$ and a thickness $t=0.2 \mathrm{~mm}$ has been modeled in this example. Fig. 3 shows its cross section that depends on the horizontal $S_{x}$ and vertical $S_{y}$ spacing between the conductors. A trivariate reduced order model is built as a function of frequency and the horizontal and vertical spacing. Table I shows their corresponding ranges.

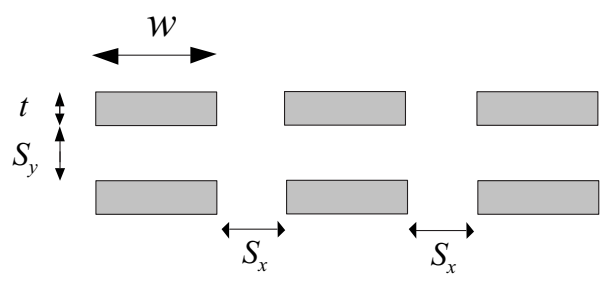

Fig. 3. Cross section of the multiconductor system. 
TABLE I

PARAMETERS OF THE MULTICONDUCTOR SYSTEM.

\begin{tabular}{|l|c|c|}
\hline Parameter & Min & Max \\
\hline Frequency (freq) & $1 \mathrm{kHz}$ & $15 \mathrm{GHz}$ \\
Horizontal spacing $\left(S_{x}\right)$ & $2 \mathrm{~mm}$ & $3 \mathrm{~mm}$ \\
Vertical spacing $\left(S_{y}\right)$ & $1 \mathrm{~mm}$ & $2 \mathrm{~mm}$ \\
\hline
\end{tabular}

The PEEC method is used to compute the $\mathbf{C}, \mathbf{G}, \mathbf{B}, \mathbf{L}$ matrices in (1a)-(1b) over an estimation grid composed of 67 scattered values of $S_{x}, S_{y}$ and a validation grid composed of 15 values of $S_{x}$ and 15 values of $S_{y}$. The order of all original PEEC models is equal to $n_{u}=702$. Then, the Laguerre-based MOR algorithm is used to build reduced models for the 67 scattered values in the design space, each with a reduced order $q=54$. These scattered points in the 2-D design space composed of the variables $\left(S_{x}, S_{y}\right)$ are chosen by a maxmin Latin hypercube design [28], enhanced by adding some data points on the boundary of the design space. A $\mathbf{Y}$-S transformation has been performed choosing $\mathrm{Z}_{0, i}=50 \Omega, i=1, \ldots, 6$, which results in a set of 67 root ROMs. The six ports of the system are defined between a conductor and the corresponding one above. A trivariate reduced model $\mathbf{S}_{r}\left(s, S_{x}, S_{y}\right)$ is obtained by multivariate simplicial interpolation of the root ROMs. Fig. 4 shows all data points in the design space selected to build (०) and validate $(\bullet)$ the parametric reduced model.

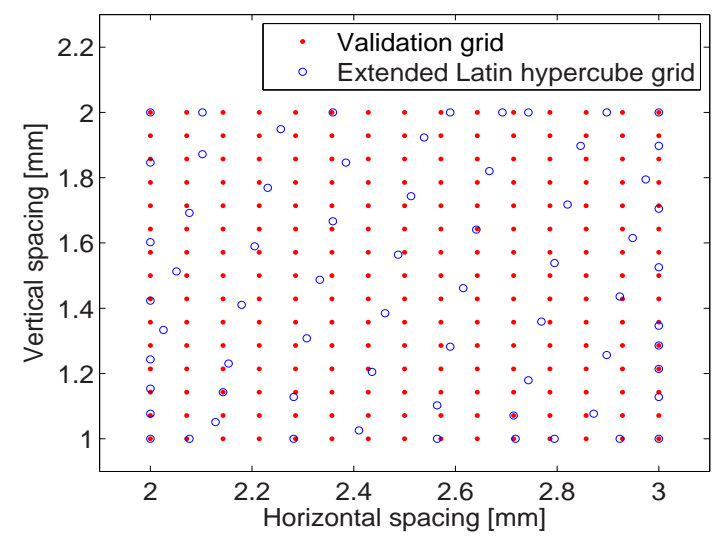

Fig. 4. Data points in the design space to build (०) and validate (•) the trivariate reduced model.

Figs. 5-6 show the magnitude of the parametric reduced model of the forward crosstalk term $\mathbf{S}_{14}\left(s, S_{x}, S_{y}\right)$ (input port of the first couple of conductors on the left and output port of the couple of conductors in the middle) obtained by multivariate simplicial interpolation for the horizontal spacing values $S_{x}=\{2,3\} \mathrm{mm}$. Fig. 7 shows the magnitude of the parametric reduced model of $\mathbf{S}_{21}\left(s, S_{x}, S_{y}\right)$ obtained by multivariate simplicial interpolation for the horizontal spacing value $S_{x}=2.5 \mathrm{~mm}$ and the vertical spacing values $S_{y}=\{1.07,1.5,1.93\} \mathrm{mm}$. These specific spacing values have not been used in the root ROM generation process. Fig. 8 shows the absolute error distribution for $\mathbf{S}_{21}\left(s, S_{x}, S_{y}\right)$ and
$\mathbf{S}_{14}\left(s, S_{x}, S_{y}\right)$ over a validation grid composed of $300 \times 15 \times 15$ ( freq, $S_{x}, S_{y}$ ) samples. The maximum absolute error of the trivariate reduced model of the $\mathbf{S}$ matrix over the validation grid is bounded by $-60.12 \mathrm{~dB}$. The parametric reduced order model describes the behavior of the system under study very accurately, while guaranteeing overall stability and passivity.

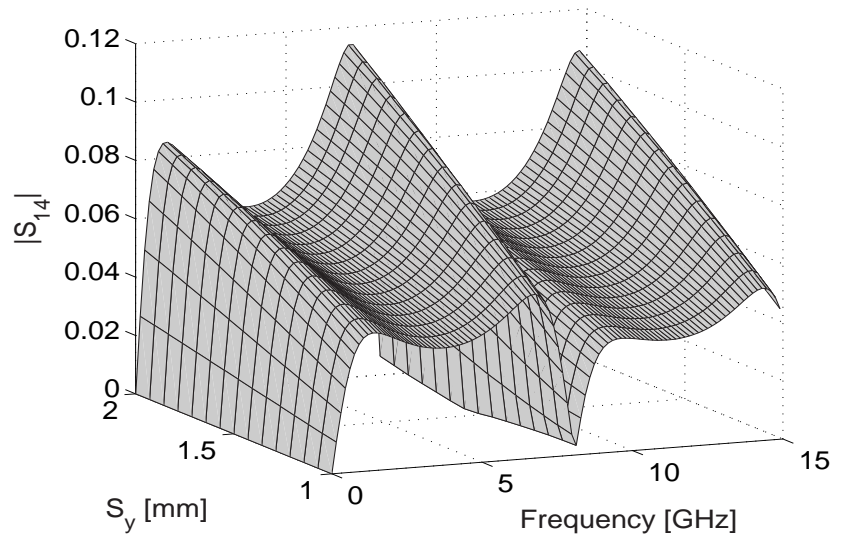

Fig. 5. Magnitude of the trivariate reduced model of $\mathbf{S}_{14}\left(s, S_{x}, S_{y}\right)\left(S_{x}=2\right.$ $\mathrm{mm})$.

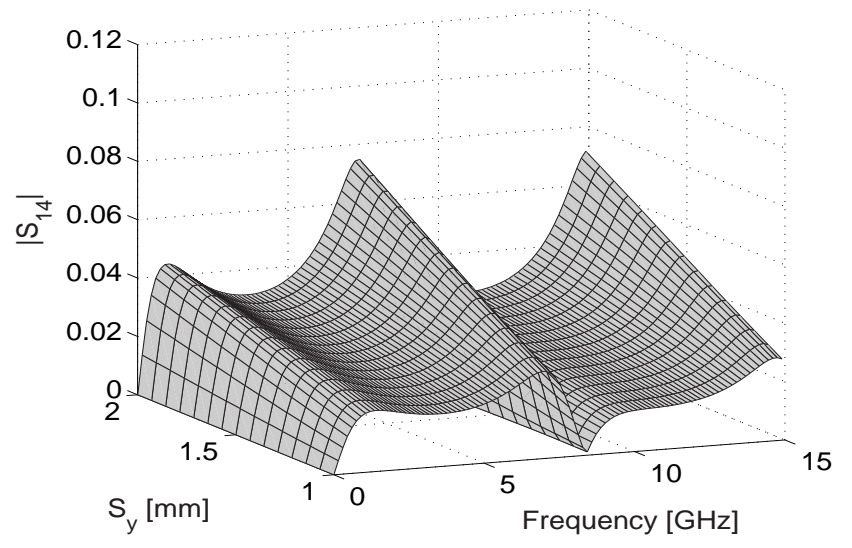

Fig. 6. Magnitude of the trivariate reduced model of $\mathbf{S}_{14}\left(s, S_{x}, S_{y}\right)$,( $S_{x}=3 \mathrm{~mm}$ ).

\section{CONCLUSiOnS}

We have presented a novel parameterized model order reduction technique applicable to the PEEC method. An efficient and reliable hybridization of traditional passivity-preserving MOR methods and interpolation schemes based on a class of positive interpolation operators is able to provide stable and passive parametric reduced order models over the design space of interest. We have proposed an interpolation scheme based on scattered design space grids that are more flexible than fully filled grids and allow the use of adaptive sampling schemes that can be useful to select a minimal and welldistributed set of points in the design space. Pertinent numer- 


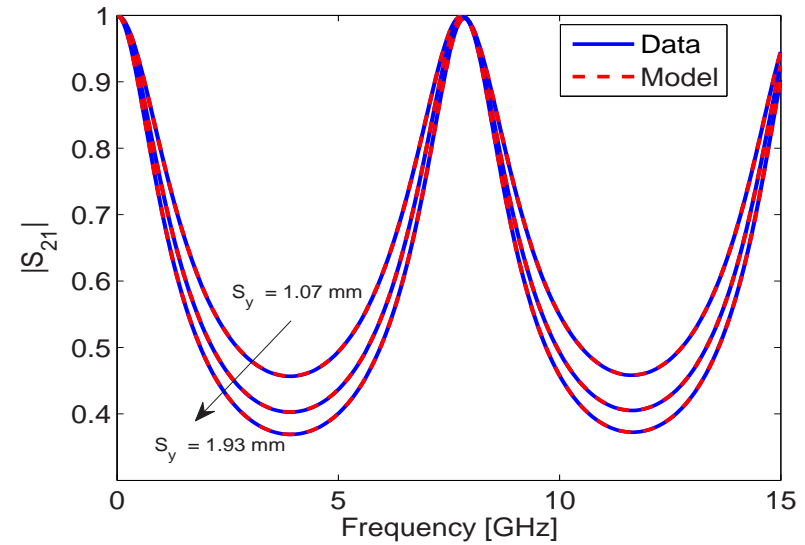

Fig. 7. Magnitude of the trivariate reduced model of $\mathbf{S}_{21}\left(s, S_{x}, S_{y}\right)\left(S_{x}=\right.$ $\left.2.5 \mathrm{~mm}, S_{y}=\{1.07,1.5,1.93\} \mathrm{mm}\right)$.

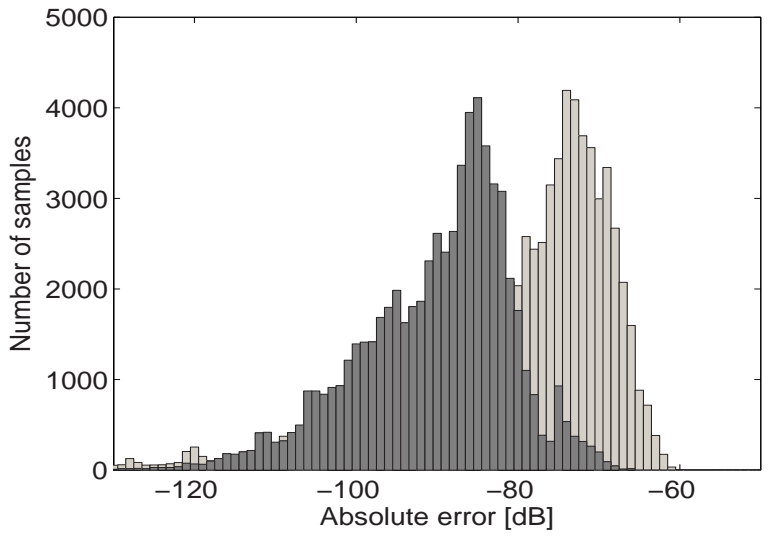

Fig. 8. Histogram : error distributions of the trivariate reduced models of $\mathbf{S}_{21}\left(s, S_{x}, S_{y}\right)$ (light grey) and $\mathbf{S}_{14}\left(s, S_{x}, S_{y}\right)$ (dark grey) over 67500 validation samples.

ical examples have validated the proposed PMOR approach on practical application cases, showing that it is able to build very accurate parametric reduced models, while guaranteeing overall stability and passivity.

\section{REFERENCES}

[1] R. F. Harrington, Field Computation by Moment Methods. New York: Macmillan, 1968.

[2] A. E. Ruehli, "Equivalent circuit models for three dimensional multiconductor systems," IEEE Trans. Microw. Theory Tech., vol. 22, no. 3, pp. 216-221, Mar. 1974.

[3] J. M. Jin, The Finite Element Method in Electromagnetics, 2nd ed. John Wiley and Sons, New York, 2002.

[4] A. Odabasioglu, M. Celik, and L. T. Pileggi, "PRIMA: passive reducedorder interconnect macromodeling algorithm," IEEE Trans. Comput.Aided Design Integr. Circuits Syst., vol. 17, no. 8, pp. 645-654, Aug. 1998.

[5] L. Knockaert and D. De Zutter, "Laguerre-SVD reduced-order modeling," IEEE Trans. Microw. Theory Tech., vol. 48, no. 9, pp. 1469-1475, Sep. 2000.

[6] A. E. Ruehli and A. C. Cangellaris, "Progress in the methodologies for the electrical modeling of interconnects and electronic packages," Proc. IEEE, vol. 89, no. 5, pp. 740-771, May 2001.
[7] W. Pinello, A. C. Cangellaris, and A. Ruehli, "Hybrid electromagnetic modeling of noise interactions in packaged electronics based on the partial-element equivalent-circuit formulation," IEEE Trans. Microw. Theory Tech., vol. 45, no. 10, pp. 1889-1896, Oct. 1997.

[8] P. Heydari and M. Pedram, "Model reduction of variable-geometry interconnects using variational spectrally-weighted balanced truncation," in Proc. IEEE/ACM Int. Conf. Comput.-Aided Des., Nov. 4-8, 2001, pp. 586-591.

[9] J. R. Phillips, "Variational interconnect analysis via PMTBR," in Proc. IEEE/ACM Int. Conf. Comput.-Aided Des., Nov. 7-11, 2004, pp. 872879.

[10] P. K. Gunupudi, R. Khazaka, M. S. Nakhla, T. Smy, and D. Celo, "Passive parameterized time-domain macromodels for high-speed transmission-line networks," IEEE Trans. Microw. Theory Tech., vol. 51, no. 12, pp. 2347-2354, Dec. 2003.

[11] L. Daniel, O. C. Siong, L. S. Chay, K. H. Lee, and J. White, "A multiparameter moment-matching model-reduction approach for generating geometrically parameterized interconnect performance models," IEEE Trans. Comput.-Aided Design Integr. Circuits Syst., vol. 23, no. 5, pp. 678-693, May 2004.

[12] Y.-T. Li, Z. Bai, Y. Su, and X. Zeng, "Model order reduction of parameterized interconnect networks via a two-directional arnoldi process," IEEE Trans. Comput.-Aided Design Integr. Circuits Syst., vol. 27, no. 9, pp. 1571-1582, Sep. 2008.

[13] G. Allasia, "Simultaneous interpolation and approximation by a class of multivariate positive operators," Numerical Algorithms, vol. 34, no. 2 , pp. 147-158, Dec. 2003.

[14] F. Ferranti, L. Knockaert, and T. Dhaene, "Parameterized S-parameter based macromodeling with guaranteed passivity," IEEE Microw. Wireless Compon. Lett., vol. 19, no. 10, pp. 608-610, Oct. 2009.

[15] P. J. Restle, A. Ruehli, S. G. Walker, G. Papadopoulos, "Full-Wave PEEC Time-Domain for the Modeling of On-Chip Interconnects," IEEE Trans. Comput.-Aided Design Integr. Circuits Syst., vol. 20, no. 7, pp. 877-887, Jul. 2001.

[16] G. Antonini, A. Orlandi, "A wavelet based time domain solution for PEEC circuits," IEEE Trans. Circuits Syst., vol. 47, no. 11, pp. 16341639, Nov. 2000.

[17] A. E. Ruehli, G. Antonini, J. Esch, J. Ekman, A. Mayo and A. Orlandi, "Non-orthogonal PEEC formulation for time and frequency domain EM and circuit modeling," IEEE Trans. Electromagn. Compat., vol. 45, no. 2, pp. 167-176, May 2003.

[18] C. Ho, A. Ruehli, P. Brennan, "The modified nodal approach to network analysis," IEEE Trans. Circuits Syst., vol. 22, no. 6, pp. 504-509, Jun. 1975.

[19] R. Rohrer, H. Nosrati, "Passivity considerations in stability studies of numerical integration algorithms," IEEE Trans. Circuits Syst., no. 9, pp. 857-866, Sep. 1981.

[20] B. D. Anderson, S. Vongpanitlerd, Network Analysis and Synthesis. Englewood Cliffs, NJ, 1973.

[21] R. W. Freund, "Krylov-subspace methods for reduced-order modeling in circuit simulation," J. Comput. Appl. Math., vol. 123, no. 1-2, pp. 395-421, 2000.

[22] C. P. Coelho, J. R. Phillips, and L. M. Silveira, "Passive constrained rational approximation algorithm using Nevanlinna-Pick interpolation," in Proc. Design, Automation and Test in Europe Conference and Exhibition, Mar. 4-8, 2002, pp. 923-930.

[23] E. W. Cheney, "Multivariate approximation theory: Selected topics," in CBMS-NSF Regional Conference Series in Applied Mathematics, vol. 51. Philadelphia, PA: SIAM, 1986.

[24] D. F. Watson, "Computing the n-dimensional Delaunay tessellation with application to Voronoi polytopes," The Computer Journal, vol. 24, no. 2, pp. 167-172, Febr. 1981.

[25] C. B. Barber, D. P. Dobkin, and H. Huhdanpaa, "The quickhull algorithm for convex hulls," ACM Trans. Math. Softw., vol. 22, no. 4, pp. 469-483, 1996.

[26] D. F. Watson, Contouring: A Guide to the Analysis and Display of Spatial Data. Pergamon Press, 1992.

[27] S. Boyd, L. El Ghaoui, E. Feron and V. Balakrishnan, Linear Matrix Inequalities in System and Control Theory. Philadelphia: SIAM, 1994.

[28] E. R. van Dam, B. Husslage, D. den Hertog, H. Melissen, "Maximin latin hypercube designs in two dimensions," Operations Research, vol. 55, no. 1 , pp. 158-169, 2007. 